\title{
Incorporação de chamote em massa cerâmica para a produção de blocos. Um estudo das propriedades físico-mecânicas
}

\section{(Grog incorporation in ceramic mass for the manufacture of bricks. A study of the physical-mechanical properties)}

\author{
F.P. Gouveia, R. M. Sposto \\ Programa de Pós-Graduação em Estruturas e Construção Civil, Departamento de Engenharia Civil e \\ Ambiental, Faculdade de Tecnologia, Universidade de Brasília - UnB, Campus Universitário Darcy Ribeiro, \\ Brasília, DF \\ gouveng@unb.br
}

\begin{abstract}
Resumo
Este trabalho apresenta um estudo das propriedades físico-mecânicas de massas cerâmicas com a incorporação de chamote visando a sua futura utilização na produção de blocos. Primeiramente foi feita a caracterização química e mineralógica, a análise granulométrica e a determinação dos limites de Atterberg da argila e do chamote. Em seguida, foram produzidos corpos de prova por extrusão, contendo argila pura e três composições de mistura, nas proporções de 5, 10, 15 e $20 \%$ em massa de chamote. A queima foi realizada em forno tipo mufla nas temperaturas de 850,950 e $1050{ }^{\circ} \mathrm{C}$. As propriedades físico-mecânicas avaliadas foram: absorção de água, porosidade aparente, retração linear e tensão de ruptura à flexão. Como resultados principais, concluiu-se que: a) a incorporação de chamote acarretou aumento na taxas de absorção de água e diminuição da retração na secagem e na queima e b) para a temperatura de $1050{ }^{\circ} \mathrm{C}$, os corpos de prova produzidos com $10 \%$ de chamote apresentaram melhores resultados para a tensão de ruptura a flexão.
\end{abstract}

Palavras-chave: chamote, cerâmica vermelha, bloco cerâmico.

Abstract

This work presents a study of the physicist-mechanical properties of ceramic masses with grog incorporated, for a future use in the ceramic bricks production. First it was made the chemical and mineralogica characterization, the grain sized analysis and the determination of the limits of Atterberg of the clay and grog. After that, bodies of test extruded had been produced, contend pure clay and three compositions of mixture, in the ratios of 5, 10, 15 and 20\% in mass of grog. The firing was carried in a muffle furnace with temperatures of the 850,950 and $1050{ }^{\circ} \mathrm{C}$. The physicist-mechanical properties evaluated were: water absorption, drying linear shrinkage, firing linear shrinkage and flexural rupture strength. As main results, we concluded that: a) the incorporation of grog caused increase the taxes of absorption of water and reduction of the retraction in the drying and in burning and b) for the temperature of $1050^{\circ} \mathrm{C}$, the produced bodies of test with $10 \%$ of grog had presented better resulted for the flexural rupture strength.

Keywords: grog, red ceramic, ceramic brick.

\section{INTRODUÇÃO}

A perda direta de blocos cerâmicos na sua produção ocorre em forma de resíduo de peças defeituosas. Esta perda é variável no Brasil, e depende de fatores tais como: matéria prima, tecnologia na fabricação e controle de qualidade adotados nas indústrias, dentre outros, e situa-se entre 2 a $10 \%$, podendo até ultrapassar o limite máximo de $10 \%$ $[1,2]$. Entre os principais defeitos observados nos blocos, que geram a perda em questão, podem ser citadas as trincas por retração, em geral derivadas do proporcionamento não adequado das argilas e do elevado teor de umidade na fabricação.

Apesar da implantação de sistemas de gestão da qualidade em muitas indústrias cerâmicas tendo como objetivo, dentre outros, a minimização de perdas no processo de produção, sabe-se que esta perda nunca será zero, ou seja, sempre existirá um percentual de perdas na indústria, denominada perda natural. Esta perda se dá de forma direta por meio do resíduo de bloco cerâmico, denominado chamote, que pode ser incorporado novamente na massa cerâmica, apresentando vantagens na melhoria dos parâmetros préqueima [3]. Porém, para o aproveitamento do chamote, com conseqüentes reações de sinterização, é necessária queima em temperaturas superiores a qual foi obtido $[2,3]$.

Este trabalho trata de um estudo experimental do efeito da incorporação do chamote em massas cerâmicas para a fabricação de blocos de vedação. Considerando-se que a 
disposição de resíduos como o chamote em aterros, lixões ou diretamente nas vias causa um impacto ambiental negativo, seu reaproveitamento é bastante oportuno, pois minimiza este impacto; além disto, há economia de matéria prima, com a geração de um processo mais sustentável na indústria cerâmica.

Oobjetivo deste trabalho é avaliaro efeito da incorporação do chamote em massas cerâmicas vermelhas dosadas em diferentes proporções, nas propriedades de retração e tensão de ruptura à flexão.

\section{MATERIAIS E MÉTODOS}

Para a realização deste trabalho, foram utilizadas argila e chamote provenientes de uma indústria cerâmica localizada em Petrolina, GO, que é considerada uma das cinco principais fornecedoras do DF. A produção mensal desta indústria é estimada em 750.000 blocos, queimados em fornos tipo intermitente com temperaturas variando de 700 a $950{ }^{\circ} \mathrm{C}$. O chamote foi obtido do descarte de blocos cerâmicos queimados a aproximadamente $800{ }^{\circ} \mathrm{C}$, conforme procedimento de coleta estabelecido [4]; a seguir, foi triturado em moinho de bolas, a partir de 8 esferas de aço com diâmetro $40 \mathrm{~mm}$ para cada $5 \mathrm{~kg}$ de amostra, com rotação $750 \mathrm{rpm}$ durante $15 \mathrm{~min}$. A argila e o chamote foram caracterizados segundo as suas composições químicas, físicas e mineralógicas. Em seguida foram determinadas as propriedades físico-mecânicas.

Para a análise da composição química da argila e do chamote utilizou-se o método da decomposição por fusão total com metaborato de lítio. A identificação dos elementos ( $\mathrm{Si}, \mathrm{Al}, \mathrm{Fe}, \mathrm{Ti}, \mathrm{Mn}, \mathrm{Ca}$ e $\mathrm{Mg}$ ) foi feita por espectrometria de emissão atômica (ICP/EAS), e do sódio (Na) e do potássio (K) por espectrometria de absorção atômica (ICP/AES). Para a caracterização mineralógica, utilizou-se a difração de raios X. Para a determinação das propriedades físicas da argila e do chamote foram realizados ensaios de análise granulométrica [5] e determinação do índice de plasticidade [6].

A argila foi inicialmente seca em estufa a $110{ }^{\circ} \mathrm{C}$ por $24 \mathrm{~h}$ e em seguida destorroada. Posteriormente separou-se a fração passante na malha 16 (abertura $1190 \mu \mathrm{m}$ ). O chamote foi peneirado em malha $20(840 \mu \mathrm{m})$. Foram selecionadas para o estudo quatro composições de argila e chamote, $5 \mathrm{CH}$, $10 \mathrm{CH}, 15 \mathrm{CH}$ e $20 \mathrm{CH}$, conforme apresentado na Tabela I. A malha 20 foi selecionada pelo fato de se buscar um melhor aproveitamento da quantidade do resíduo.

Foram produzidos 60 corpos de prova, 4 para cada
Tabela I - Composições investigadas (\% em massa). [Table I - Investigated compositions (mass \%).]

\begin{tabular}{cccccc}
\hline $\begin{array}{c}\text { Matéria } \\
\text { Prima }\end{array}$ & \multicolumn{5}{c}{ Composição } \\
\hline & ARG & $5 \mathrm{CH}$ & $10 \mathrm{CH}$ & $15 \mathrm{CH}$ & $20 \mathrm{CH}$ \\
Argila & 100 & 95 & 90 & 85 & 80 \\
Chamote & 0 & 5 & 10 & 15 & 20 \\
\hline
\end{tabular}

composição argila-chamote, nas dimensões 100 x 30 x 13,8 $\mathrm{mm}^{3}$ (comprimento, largura e altura). A homogeneização das massas foi feita de forma manual. A conformação dos corpos de prova foi realizada por extrusão em maromba a vácuo MVIG-O5. Os corpos de prova foram levados à secagem natural por $48 \mathrm{~h}$, seguida de secagem em estufa a $105-110^{\circ} \mathrm{C}$ por $24 \mathrm{~h}$. Para a etapa de queima utilizou-se forno elétrico tipo mufla (faixa de trabalho 800 a $1200{ }^{\circ} \mathrm{C}$ ). Os corpos de prova foram submetidos a três temperaturas de queima: 850 , 950 e $1050^{\circ} \mathrm{C}$. Em seguida foram determinadas as seguintes propriedades: retração linear, absorção de água, porosidade aparente e resistência à flexão. A retração linear foi obtida a partir do comprimento dos corpos de prova iniciais e finais após a secagem e a queima, para as diferentes temperaturas estabelecidas. A absorção de água e a porosidade aparente foram determinadas a partir dos procedimentos estabelecidos em [7]. A tensão de ruptura à flexão foi determinada a partir de 3 pontos [8]. Utilizou-se uma máquina de ensaio universal de laboratório MTS 810, programada para operar com taxa de carregamento de $2 \mathrm{~mm} / \mathrm{min}$. A distancia entre os apoios foi fixada em $8 \mathrm{~cm}$.

\section{RESULTADOS E DISCUSSÃO}

\section{Composição química}

Os resultados obtidos são apresentados na Tabela II.

Observou-se que para ambas as amostras analisadas o principal constituinte é sílica $\left(\mathrm{SiO}_{2}\right)$, com percentuais de $54,3 \%$ e $64,06 \%$ para a argila e o chamote, respectivamente. Quanto à alumina $\left(\mathrm{Al}_{2} \mathrm{O}_{3}\right)$ e à hematita $\left(\mathrm{Fe}_{2} \mathrm{O}_{3}\right)$, os valores encontrados nas amostras foram próximos. Foram identificados baixos teores de óxidos alcalinos $\left(\mathrm{K}_{2} \mathrm{O}\right.$ e $\left.\mathrm{Na}_{2} \mathrm{O}\right)$ e alcalinos terrosos $(\mathrm{MgO}$ e $\mathrm{CaO})$. Em relação à perda ao fogo o chamote apresentou valor percentual menor que a argila, o que pode ser explicado devido ao processo de queima anterior. Por meio da análise química pode-se notar a similaridade dos elementos da argila e do resíduo.

Tabela II - Análise química da argila e do chamote, em \%.

[Table II - Chemical analysis of clay and grog \%.]

\begin{tabular}{cccccccccccc}
\hline AMOSTRA & $\mathrm{SiO}_{2}$ & $\mathrm{Al}_{2} \mathrm{O}_{3}$ & $\mathrm{Fe}_{2} \mathrm{O}_{3}$ & $\mathrm{TiO}_{2}$ & $\mathrm{MnO}$ & $\mathrm{MgO}$ & $\mathrm{CaO}$ & $\mathrm{Na}_{2} \mathrm{O}$ & $\mathrm{K}_{2} \mathrm{O}$ & P.F & Total \\
\hline Argila & 54,3 & 19,97 & 9,47 & 1,45 & 0,22 & 1,04 & 1,47 & 0,29 & 0,91 & 10,44 & 99,56 \\
Chamote & 64,06 & 17,62 & 10,83 & 1,67 & 0,13 & 0,77 & 0,61 & 0,17 & 1,23 & 2,82 & 99,91 \\
\hline
\end{tabular}




\section{Granulometria}

NaFig. 1 sãoapresentadas as distribuições granulométricas das partículas para a argila e o chamote.

Observa-se para a argila utilizada o predomínio da granulação argilo-arenosa, com um percentual de 41,1\% de argila e 59,4\% de silte e areia (36,7 e 21,6\%, respectivamente para areia e silte). Os percentuais estão fora do recomendado, que é de $60 \%$ para a argila e $40 \%$ para o silte e a areia [9], indicando uma proporção de argila inferior e uma proporção de silte elevada. Para o chamote ocorreu a predominância da fração de areia, com valor de 51,1\%, um baixo percentual de argila, com valor de $1,7 \%$ e um considerável percentual de pedregulho de $20 \%$, o que demonstra que a sua trituração não foi eficiente. A provável justificativa para esse inconveniente seria o procedimento adotado para cominuir o material, no que diz respeito ao número de rotações do equipamento, o número de esferas e o tempo utilizado para o processo

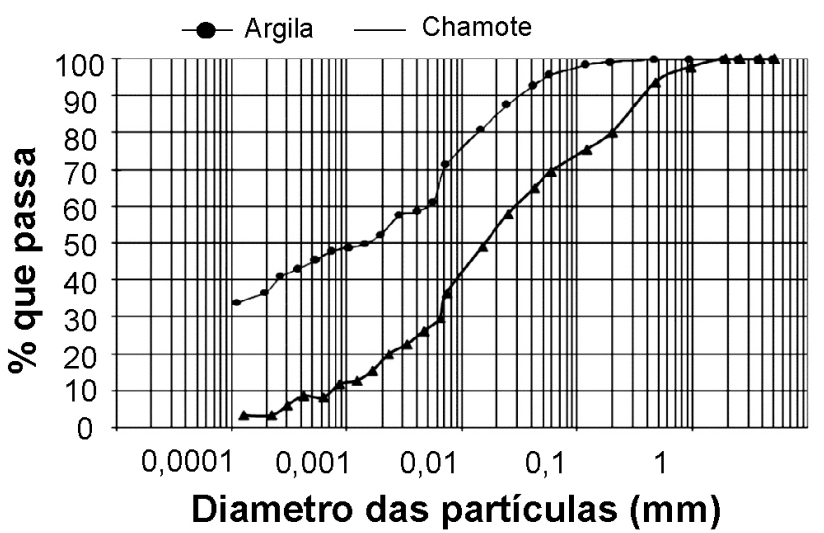

Figura 1: Curvas de distribuição de tamanho de partícula para a argila e o chamote.

[Figure 1: Particle size distribution curves of clay and grog.]

\section{Limites de Atterberg}

Obteve-se para a argila um valor de LP de $28 \%$ e LL 49\%, o que corresponde a um IP $21 \%$. Por meio do IP pôde-se concluir que argila é altamente plástica, porém dentro do limite sugerido de 10 a $35 \%$ [10]. O chamote não apresentou plasticidade, podendo ser considerado como um material não plástico. E para as composições $5 \mathrm{CH}, 10 \mathrm{CH}, 15 \mathrm{CH}$ e $20 \mathrm{CH}$ obtiveram-se valores de LP iguais a 29, 26, 27 e 28, respectivamente; estes valores apresentaram pequena variação em relação à $\mathrm{ARG}$. $\mathrm{O}$ menor LP foi obtido para $\mathbf{1 0 C H}$, implicando em uma menor quantidade de água para conformação.

\section{Composição mineralógica}

Na Fig. 2 é apresentada a composição mineralógica para o caso da argila com os seguintes minerais: quartzo, ilita, caulinita, pirofilita, hematita, rutilo e microclíneo.

$\mathrm{Na}$ Fig. 3 é apresentado o difratograma de raios $\mathrm{X}$ do chamote com a identificação dos seguintes minerais:

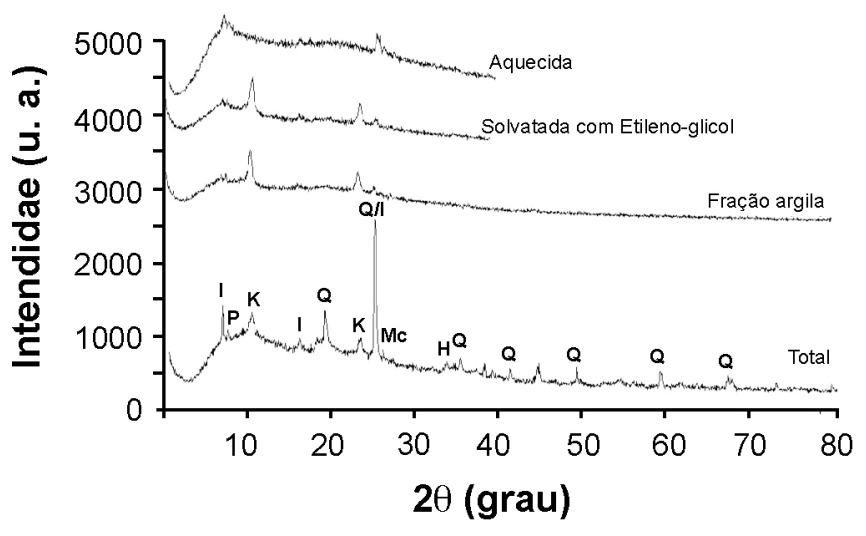

Figura 2: Difratogramas de raios $\mathrm{X}$ da argila identificando as reflexões características dos minerais: Q- quartzo, K- caulinita, Iilita, H- hematita, P- pirofilita, Mc- microclíneo.

[Figure 2: X-ray diffraction patterns of clay, identifying the reflections characteristics of minerals: $Q$ - quartz, $K$ - kaolinite, $I$ illite, $H$-hematite, $P$ - pyrophilite, Mc-microcline.]

quartzo, ilita, (no lugar de mica), hematita, rutilo e diásporo. A presença de mica se deve à modificaçao da estrutura da ilita com a queima.

$\mathrm{Na}$ amostra total identificou-se quartzo como constituinte predominante, como constituinte menor e hematita, diásporo e rutilo como minerais traços. Vale destacar que a caulinita identificada na amostra de argila não esteve presente no chamote.

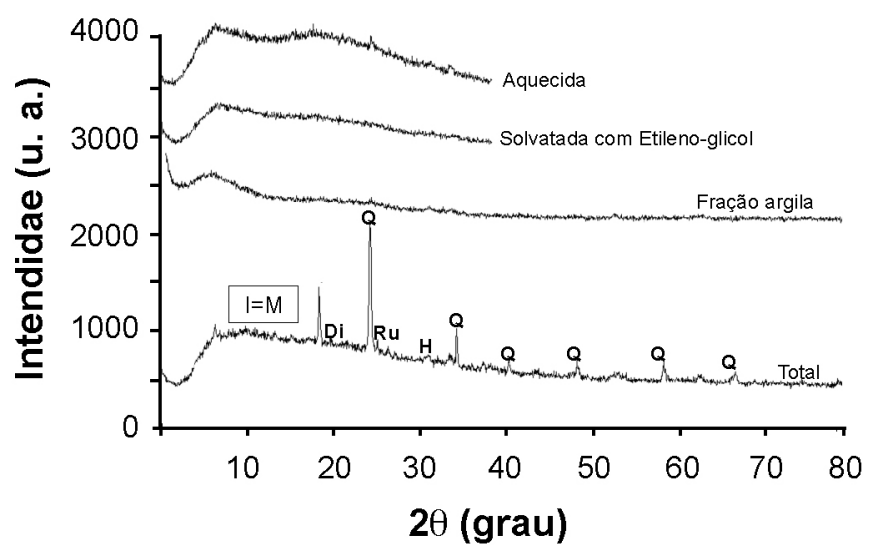

Figura 3: Difratogramas de raios $\mathrm{X}$ do chamote identificado as reflexões características dis minerais: Q - quartzo, I - ilita, H hemati9ta, RU - rutilo, Di - diásporo.

[Figure 3: X-ray diffraction patterns of clay, identifying the reflections characteristics of minerals: $Q$ - quartz, $K$ - kaolinite, $I$ illite, $H$ - hematite, $R u$ - rulite, $D i$ - diapore.]

\section{Propriedades físico-mecânicas}

Na Tabela III são apresentados os resultados da retração linear de secagem (RLS) para as composições analisadas.

Os resultados indicaram que na amostra estudada a incorporação de chamote gerou uma tendência de diminuição da RLS, com o menor percentual para $20 \mathrm{CH}$. Isto pode ser 
Tabela III - Retração linear de secagem dos corpos cerâmicos.

[Table III - Linear shrinkage of ceramic pieces.]

\begin{tabular}{cc}
\hline Composições & $\begin{array}{c}\text { RLS } \\
(\%)\end{array}$ \\
\hline ARG & 3,50 \\
$5 \mathrm{CH}$ & 3,47 \\
$10 \mathrm{CH}$ & 3,07 \\
$15 \mathrm{CH}$ & 3,56 \\
$20 \mathrm{CH}$ & 2,97 \\
\hline
\end{tabular}

explicado pelo efeito da adição de não-plásticos (caso do chamote) às argilas reduzir as contrações sofridas pela massa, melhorando este parâmetro de pré-queima das composições, e corroborando com outros estudos da incorporação de chamote em massas cerâmicas [3].

Na Fig. 4 é apresentada a curva de gresificação para ARG e $5 \mathrm{CH}, 10 \mathrm{CH}, 15 \mathrm{CH}$ e $20 \mathrm{CH}$.

A partir da Fig. 4, que relaciona a absorção de água (AA) e a retração linear de queima (RLQ) em função da temperatura, pôde-se constatar na amostra considerada o decréscimo de AA com o aumento de temperatura e o aumento de AA com a incorporação de chamote, fato já constatado por [11]. A $850{ }^{\circ} \mathrm{C}$ todas as composições apresentaram aumento de AA, sendo o maior valor para 20CH. A $950{ }^{\circ} \mathrm{C}$ pôde-se observar o aumento de AA para $15 \mathrm{CH}$ e $20 \mathrm{CH}$. E a $1050{ }^{\circ} \mathrm{C}, 10 \mathrm{CH}, 15 \mathrm{CH}$ e $20 \mathrm{CH}$ apresentaram aumento de AA, com o maior valor para a composição $20 \mathrm{CH}$; estes valores estão dentro do intervalo de 0 a $22 \%$, indicado para AA para blocos cerâmicos furados [12]. Em relação à RLS obtiveram-se para ARG os maiores valores percentuais iguais a 3,$28 ; 3,26$ e 3,31 , correspondentes as temperaturas de $850{ }^{\circ} \mathrm{C}, 950{ }^{\circ} \mathrm{C}$ e 1050 ${ }^{\circ} \mathrm{C}$. Observou-se que, à medida que o chamote foi sendo incorporado, ocorreu o decréscimo dos valores obtidos para as três temperaturas, exceto no caso de $20 \mathrm{CH}$, na

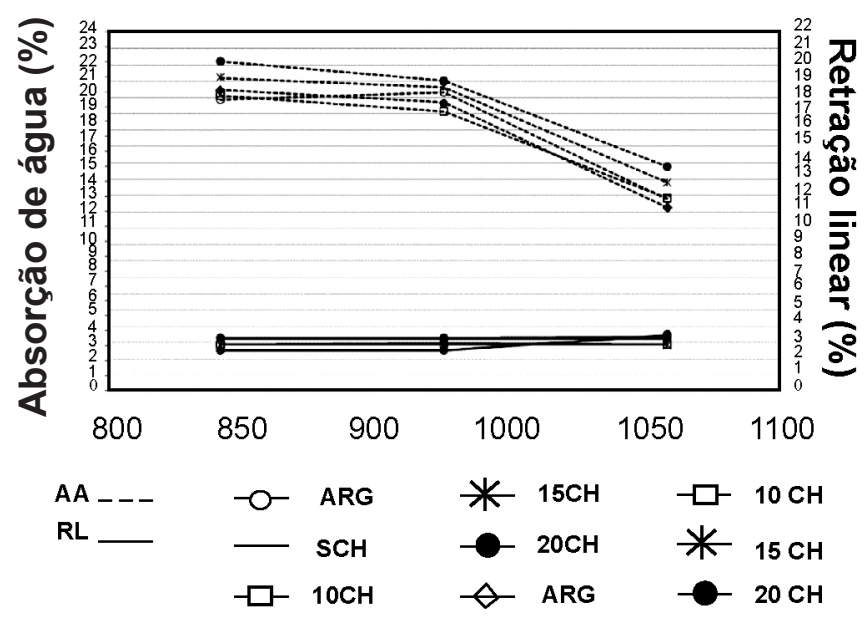

Figura 4: Curva de gresificação das composições estudadas. [Figure 4: Vitrification curve of the studied compositions.] temperatura de $1050{ }^{\circ} \mathrm{C}$. Este comportamento é de extrema importância na produção de elementos cerâmicos, uma vez que potencializa o controle da retração [13].

Vale ressaltar que a porosidade aparente das composições também foi investigada e o maior valor foi obtido para $20 \mathrm{CH}$, igual a $36,4 \%$. As composições ARG indicaram menores percentuais com um mínimo de 21,6\% para $1050^{\circ} \mathrm{C}$. Os valores das composições intermediárias variaram entre os percentuais mínimos e máximos citados. Não se pôde, porém, concluir sobre o efeito do chamote no empacotamento, uma vez que a densidade aparente a seco das composições não foi investigada.

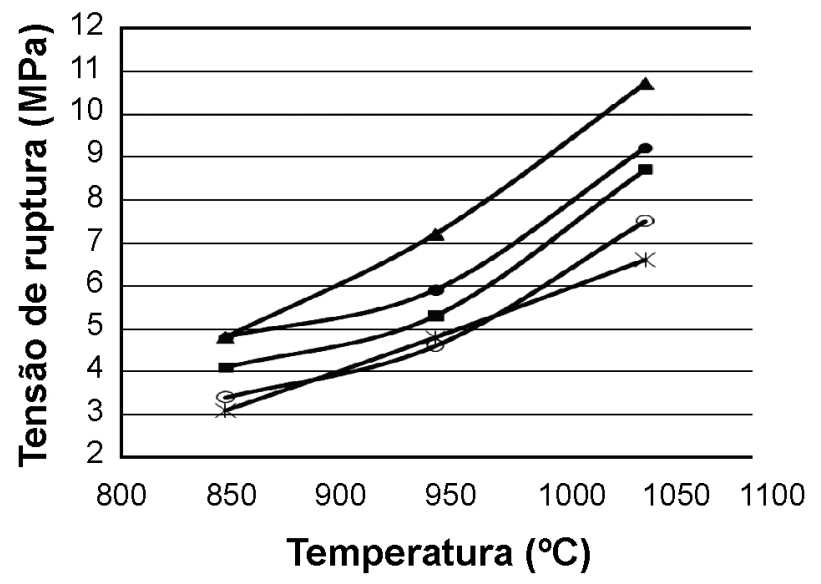

ARG

$5 \mathrm{CH}$

$10 \mathrm{CH} \odot 15 \mathrm{CH} \rightarrow 20 \mathrm{CH}$

Figura 5: Tensões de ruptura à flexão dos corpos cerâmicos. [Figure 5: Flexural rupture strength of ceramic pieces.]

\section{Tensão de ruptura à flexão}

Na Fig. 5 são apresentados os resultados obtidos da tensão de ruptura à flexão dos corpos cerâmicos sinterizados para cada composição.

Em relação aos valores médios de tensão de ruptura observou-se que: a $850{ }^{\circ} \mathrm{C}$ ARG e $10 \mathrm{CH}$ apresentaram os maiores valores médios, iguais a 4,8 $\mathrm{MPa}$, e o desvio padrão foi de 0,5 e 0,8 respectivamente; a $950{ }^{\circ} \mathrm{C}$, ARG e $10 \mathrm{CH}$ apresentaram os maiores valores iguais a 5,9 e 7,2 MPa e desvio padrão de 0,3 e 0,6 respectivamente e a 1050 ${ }^{\circ} \mathrm{C}$, ARG e $10 \mathrm{CH}$ também apresentaram os maiores valores de tensão, iguais a 9,2 e 10,7 MPa com desvio padrão de 1,1 e 0,7 respectivamente. A $1050{ }^{\circ} \mathrm{C}$ todos os corpos de prova apresentaram valores superiores a 5,5 $\mathrm{MPa}$, valor estipulado para a tensão de ruptura após a queima na produção de blocos cerâmicos [14]. Isto sugere a utilização de chamote para esta temperatura. Ainda, constatou-se que à medida que se eleva a temperatura há um incremento no valor da tensão de ruptura, observando-se que a queima é um processo que envolve o aquecimento do material a altas temperaturas de forma a promover a vitrificação das partículas. Os melhores resultados foram obtidos para 
$10 \mathrm{CH}$. Para $15 \mathrm{CH}$ e $20 \mathrm{CH}$ ocorreu redução nos valores de resistência.

\section{CONCLUSÕES}

A argila utilizada apresentou alta plasticidade, o que pode ser corrigido pela incorporação de $10 \%$ de chamote, $10 \mathrm{CH}$, menor valor obtido para o limite de plasticidade. Entretanto, de uma forma geral, a incorporação do chamote não alterou significativamente os limites de plasticidade das composições analisadas. A incorporação de chamote promoveu uma diminuição na retração linear de secagem e de queima, o que pode ser benéfico para a utilização na produção de blocos cerâmicos, possibilitando a diminuição de ocorrência de trincas por retração nos blocos. Os valores da absorção de água das amostras diminuíram com o aumento da temperatura e aumentaram gradativamente com a adição de chamote; entretanto, permaneceram dentro dos limites recomendados por normas para blocos cerâmicos. Observa-se que a tensão de ruptura à flexão dos corpos de prova aumentou em função da elevação da temperatura de queima, com valores máximos para $1050{ }^{\circ} \mathrm{C}$. Dentre as composições analisadas, destaca-se que a incorporação de $10 \%$ de chamote, $10 \mathrm{CH}$, apresentou os maiores valores de resistência para as três temperaturas.

\section{REFERÊNCIAS}

[1] A. T. Mafra, Proposta de indicadores de desempenho para a indústria cerâmica vermelha, Diss. Mestrado, Universidade Federal de Santa Catarina, Florianópolis, SC (1999) 113.

[2] N. de S. Campelo, M. R. Morais, A. F. Aragão, E. M. Cabral, E. de P. Rabelo, S. C. Pinheiro, O.A. Paiva, Cerâmica
Industrial 11, 1 (2006) 44.

[3] C. M. S. Vieira, E. T. A. de Sousa, S. N. Monteiro, Cerâmica 50, 315 (2004) 254.

[4] ABNT - Associação Brasileira de Normas Técnicas, NBR 10007, Amostragem de resíduos sólidos, Rio de Janeiro, RJ (2004).

[5] ABNT - Associação Brasileira de Normas Técnicas, NBR 7181, Solo - Análise granulométrica: Método de ensaio, Rio de Janeiro, RJ (1984).

[6] ABNT - Associação Brasileira de Normas Técnicas, NBR 7180, Solo - Determinação do limite de plasticidade: Método de ensaio, Rio de Janeiro, RJ (1984).

[7] ABNT - Associação Brasileira de Normas Técnicas, NBR 6458, Grãos de pedregulho retidos na peneira de 4,8 mm - Determinação da massa específica, da massa específica aparente e da absorção de água, Rio de Janeiro, RJ (1984).

[8] ABNT - Associação Brasileira de Normas Técnicas, NBR 13816, Placas cerâmicas para revestimento -Especificação e método de ensaios, Rio de Janeiro, RJ (1997).

[9] E. G. R. Petrucci, Materiais de construção. 10a Ed., Globo, S. Paulo, SP (1998) 9.

[10] H. P. Caputo, Mecânica dos solos e suas aplicações, Vol

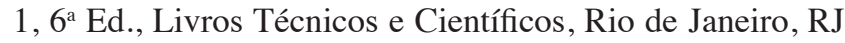
(1988) 56.

[11] I. Demir, M. Orhan, Building Environment 38, 12 (2003) 1451.

[12] ABNT - Associação Brasileira de Normas Técnicas, NBR 15270-1, Blocos cerâmicos para alvenaria de vedação - Terminologia e requisitos, Rio de Janeiro, RJ (2005).

[13] S. Pracidelli, F. G. Melchiades, Cerâmica Industrial 2, 1-2 (1997) 31.

[14] P. Souza Santos, Ciência e Tecnologia de argilas, 2 ${ }^{a}$ Ed., Edgard Blücher, S. Paulo, SP (1989) 397.

(Rec. 09/09/2008, Rev. 22/12/2008, Ac. 15/01/2009) 